\title{
Analysis of Bottom-Track and Compass Error in a Self-Contained Acoustic Doppler Diver Navigation Console
}

\author{
JAMES L. HENCH \\ Marine Laboratory, Nicholas School of the Environment, Duke University, Beaufort, North Carolina \\ JOHANNA H. ROSMAN \\ Institute of Marine Sciences, University of North Carolina at Chapel Hill, Morehead City, North Carolina
}

(Manuscript received 2 November 2009, in final form 16 February 2010)

\begin{abstract}
The Cobra-Tac (Teledyne RD Instruments) is a self-contained diver navigation system based on acoustic Doppler velocity log (DVL) technology that uses dead reckoning to compute displacements from a known starting point. The navigational accuracy of the system was evaluated using a series of field tests with known solutions. Initial test data had an obvious directional bias in the navigation measurements, with positional errors as large as $5 \%$ of the track length. An analysis of this error showed that the DVL speed measurements were extremely accurate and that most of the position error could be explained by heading-dependent compass error. Compass error versus heading curves were different depending on whether a given route was mapped in a clockwise or counterclockwise direction, and further testing indicated that this was due to a combination of local magnetic field anomalies at the test site and instrument tilt. A postprocessing correction procedure, based on a one-cycle compass error model, was derived that significantly improves Cobra-Tac position estimates. After correction, position errors were well within manufacturer specifications. Further tests using new firmware that permitted in situ underwater compass calibration gave position errors of less than $1 \%$ of the track length. This level of accuracy should be sufficient for a wide range of scientific applications. Collectively, the test results and analyses indicate that Cobra-Tac can give very accurate navigation results, but the accuracy depends strongly on compass calibration and diver skill with the system.
\end{abstract}

\section{Introduction}

For many underwater scientific studies, scuba diving is used to directly observe and quantify marine systems or to deploy and recover instrumentation. Accurate underwater navigation can be the limiting step, particularly in low-visibility environments or in areas without obvious underwater landmarks. Even under optimal diving conditions, accurate and repeatable underwater diver navigation may be required. Traditionally, divers have used simple methods for navigation, such as hand-held compasses, to determine direction and counting kick cycles to estimate distances (Joiner 2001). Benthic mapping has typically been done with manual transect tapes combined

Corresponding author address: James L. Hench, Marine Laboratory, Duke University, 135 Duke Marine Lab Rd., Beaufort, NC 28516.

E-mail: jim.hench@duke.edu with underwater notes (Merifield and Rosencrantz 1966; Joiner 2001). For many tasks traditional methods are sufficient, but for more complex work such as bathymetric mapping, benthic biota surveys, and the positioning of sensor arrays they can be prohibitively time consuming or inaccurate.

More recently, diver navigation systems have been developed that determine diver locations using triangulation from an array of acoustic transponders (e.g., Newborough and Woodward 1999). However, there may be situations where deploying and surveying in transponders is impractical or undesirable. Moreover, navigation using acoustic transponders requires that divers work within the line of sight of the sound sources and there can be multipath errors in position calculations. Ideally, a diver navigation system would be self-contained and not require transponders. The first commercially available autonomous diver navigation system is the Cobra-Tac (Teledyne RD Instruments 2007). The system is based on 


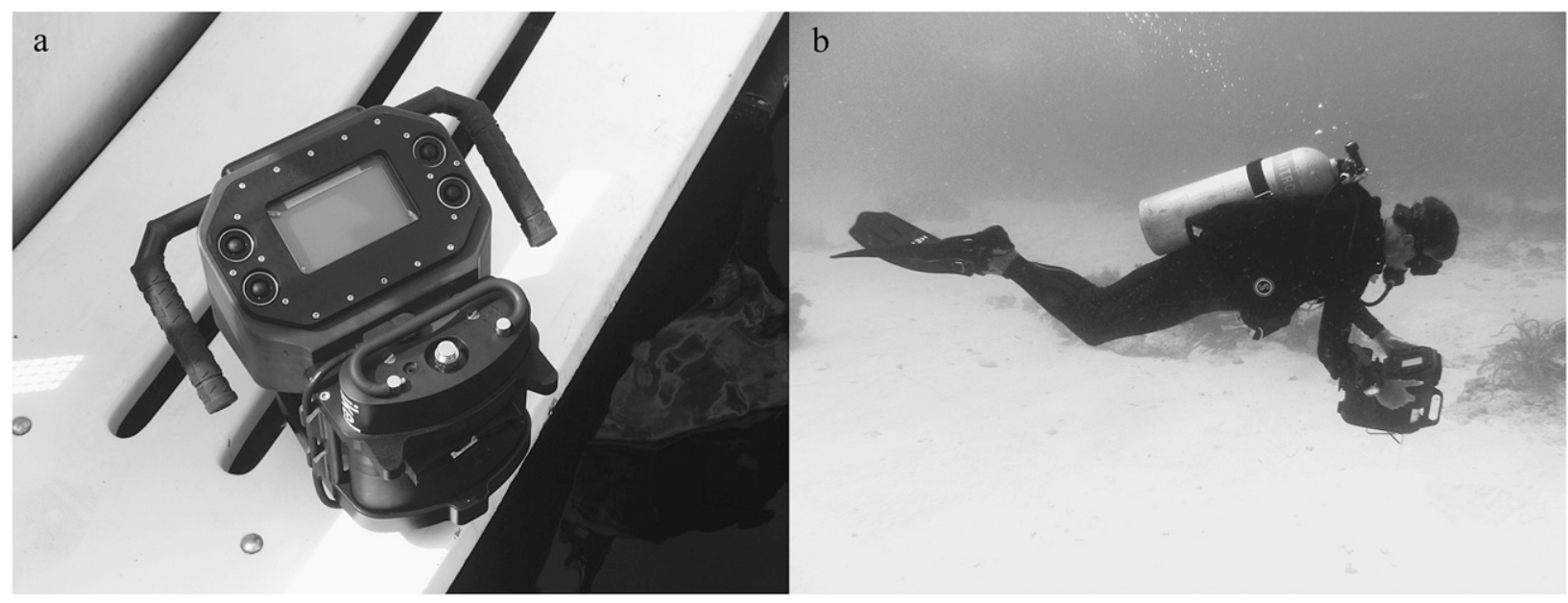

FIG. 1. (a) The Cobra-Tac diver navigation console and (b) a scuba diver navigating with a Cobra-Tac near a coral patch reef.

Doppler velocity log (DVL) technology in which dead reckoning is used to compute displacements from a known starting point using bottom tracking, similar to that used in a shipboard acoustic Doppler current profiler (ADCP) or autonomous underwater vehicle (AUV).

The Cobra-Tac was originally developed for military applications, but it could be useful for scientific purposes. To our knowledge, there are no published studies using a Cobra-Tac, so the immediate questions are, 1) how accurate is the instrument's navigation and 2) could it be used for science? Previous work with shipboard ADCPs suggests that, although speed estimates from the bottom track are accurate, headings from the internal fluxgate compass can contain significant errors (Joyce 1989; Trump and Marmorino 1997; Fong and Monismith 2004). Moreover, the compass heading errors are typically directionally dependent. Work with shipboard ADCPs has shown that heading errors can be corrected using an external navigation source such as a gyrocompass (Joyce 1989) or differential GPS (Griffiths 1994; Munchow et al. 1995). AUV compass errors can be similarly corrected in real time using transponder baseline fixes to supplement the bottom-track navigation (Moline et al. 2005). Because the Cobra-Tac diver navigation system is completely selfcontained, none of these supplemental navigation corrections are available.

In this paper, we summarize the results of a set of field tests in which the Cobra-Tac was used to map out known paths. The tests show that navigation error results primarily from heading-dependent error in the Cobra-Tac internal compass. An analysis of this error is presented and a postprocessing correction method is proposed that significantly improves Cobra-Tac positional records. Finally, errors before and after the correction are compared with accuracies specified by the manufacturer.

\section{Methods}

\section{a. Description of Cobra-Tac}

Cobra-Tac (Teledyne RD Instruments, Poway, California) is a self-contained diver navigation console consisting of a DVL, battery pack, and real-time liquid crystal display (LCD) of position and navigation information (see Fig. 1). Firmware version 30.10 was used for the majority of the tests. The DVL operates at $1.2 \mathrm{MHz}$ with four orthogonal beams set at a $30^{\circ}$ angle from vertical. The beams are numbered in clockwise $(\mathrm{CW})$ order: $3,2,4$, and 1 . The angle between the front of the instrument (intended swim direction) and the beam 3 transducer is $18.4^{\circ}$ (also known as the beam 3 misalignment).

The Cobra-Tac records time, depth, altitude above bottom, temperature, bottom-track speed and direction, heading, pitch, and roll. In its present form, the CobraTac does not measure water velocities. The nominal ping rate is $5.5 \mathrm{~Hz}$; however, the instrument pings as fast as it can emit and process, and this can be slightly faster or slower than the nominal rate depending on the distance to the bottom. All parameters are recorded at the ping rate. The bottom track works between 0.5 and $30 \mathrm{~m}$ above bottom, compatible with typical scuba diving profiles. If the bottom track is lost, Cobra-Tac continues to compute positions for up to $5 \mathrm{~s}$, based on dead reckoning from the last good speed and direction. After this time, zero velocity (i.e., stationary diver) is assumed until the next good ping is obtained.

Direction is determined using an internal fluxgate compass that the manufacturer specifies as having $2^{\circ}$ accuracy, $0.01^{\circ}$ resolution, and $0.5^{\circ}$ root-mean-square (rms) noise (Teledyne RD Instruments 2007). The tilt sensor is filled with liquid electrolyte and four electrodes sense fluid movement along two perpendicular axes as the sensor 
angle changes with respect to vertical. The tilt sensor accuracy is $0.5^{\circ}$, the resolution is $0.01^{\circ}$, and rms noise is $0.35^{\circ}$ (Teledyne RD Instruments 2007). Immediately prior to the field tests, the Cobra-Tac fluxgate compass was bench calibrated (following Teledyne RD Instruments 2007) to remove hard- and soft-iron effects to within $0.6^{\circ}$.

\section{b. Idealized path tests}

Field tests were conducted at Conch Reef, about $10 \mathrm{~km}$ offshore from Key Largo, Florida $\left(24^{\circ} 57.0^{\prime} \mathrm{N}\right.$, $80^{\circ} 27.3^{\prime} \mathrm{W}$; magnetic declination $5^{\circ} 27^{\prime} \mathrm{W}$ ). Four idealized test paths were setup on a flat sandy area and on a rough coral reef area that had highly irregular (about $1 \mathrm{~m}$ high) bottom relief. In these tests, the diver swam with the instrument $1 \mathrm{~m}$ above bottom. The first test consisted of a series of reciprocal course out and back swims along a 14-m-long ground line oriented northeastsouthwest, followed by out and back swims along a ground line oriented perpendicular to the original path. In the second test, repeated circle sweeps were made with an 8-m line tied between the Cobra-Tac and a $25-\mathrm{kg}$ lead weight buried at the center, thus the heading changed continuously. The effective radius was about $7.8 \mathrm{~m}$ due to the diver elevation above the seafloor. The diver made repeated laps in both the $\mathrm{CW}$ and counterclockwise (CC) directions, starting and ending at the same point in the circle. In the third test, a box with 14-m sides was measured with transect tapes and compasses and marked with ground lines. As in the circles test, the diver made repeated circuits around the box, starting and ending at the same corner. In the final test, "random swimming" was done within the box described above by starting and ending at one corner but never leaving the box interior. Pitch and roll measurements indicated that the diver was able to keep the instrument reasonably level during the above tests: pitch was $-3.2^{\circ} \pm 2.5^{\circ}$ and roll was $0.5^{\circ} \pm 2.4^{\circ}$ (where \pm indicates one standard deviation about the mean).

\section{c. Dependence of error on pitch, roll, and height above bottom}

Another series of tests were performed to look at the effects of pitch, roll, and height above bottom on CobraTac accuracy. These tests were performed over a flat sandy bottom in Moorea, French Polynesia $\left(17^{\circ} 29.0^{\prime} \mathrm{S}\right.$, $149^{\circ} 50.3^{\prime} \mathrm{W}$; magnetic declination $12^{\circ} 48^{\prime} \mathrm{E}$ ). This site was chosen because it was known to be far from any ferromagnetic materials. The diver swam repeatedly around an 8-m-radius circular path as before. In the first test, the instrument was held as level as possible at a height of $1 \mathrm{~m}$ above bottom. In the second and third tests, Cobra-Tac was intentionally tilted with a constant pitch and roll,
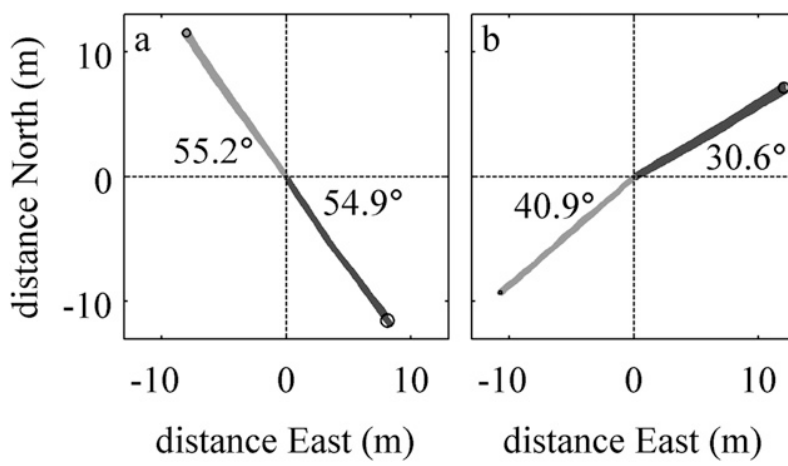

FIG. 2. Summary of reciprocal course data: (a) six out and back tests in approximately SE-NW directions and (b) four out and back tests in approximately NE-SW directions. Dark gray indicates the outbound navigation fixes and light gray indicates the return navigation fixes. Black circles show $95 \%$ confidence area of the ending point for the repeated tests. Degree values are the mean navigation direction for the repeated tests in each direction; the reciprocal directions would be equal if the navigation was perfect.

respectively. In the final test, the instrument was held as level as possible at $2 \mathrm{~m}$ above bottom.

\section{d. Idealized tests with in situ compass calibration}

In response to the results of the initial tests, described below, Teledyne RD Instruments provided modified firmware (version 30.10c) that permitted in situ compass calibration by the diver while underwater. It was expected that this would provide an optimal compass calibration. The circle test described in section $2 \mathrm{c}$ was repeated 3 times at the Moorea site with the instrument held as level as possible at $1 \mathrm{~m}$ above bottom. A new in situ calibration was performed immediately prior to each test.

\section{Results and analysis}

\section{a. Idealized path results}

The first test, out and back swims along a 14-m ground line over a flat sandy bottom, indicated that Cobra-Tac measurements of distance are accurate but that the directional error can be quite large (Fig. 2). The length of the path determined from Cobra-Tac measurements agreed with the length of the ground line measured using transect tapes within $0.2 \mathrm{~m}$, or $4 \%$ of the pathlength. As the accuracy of the diver's judgment of the beginning and end of the lines while swimming is probably no better than $0.2 \mathrm{~m}$, the average DVL bottom-track speed was found to be correct to within testable limits. The discrepancy between the angle of out and back paths was $10.3^{\circ}$ for the northeast and southwest swims, whereas the discrepancy was much smaller $\left(0.3^{\circ}\right)$ for the northwest and southeast swims. The directional discrepancies were 

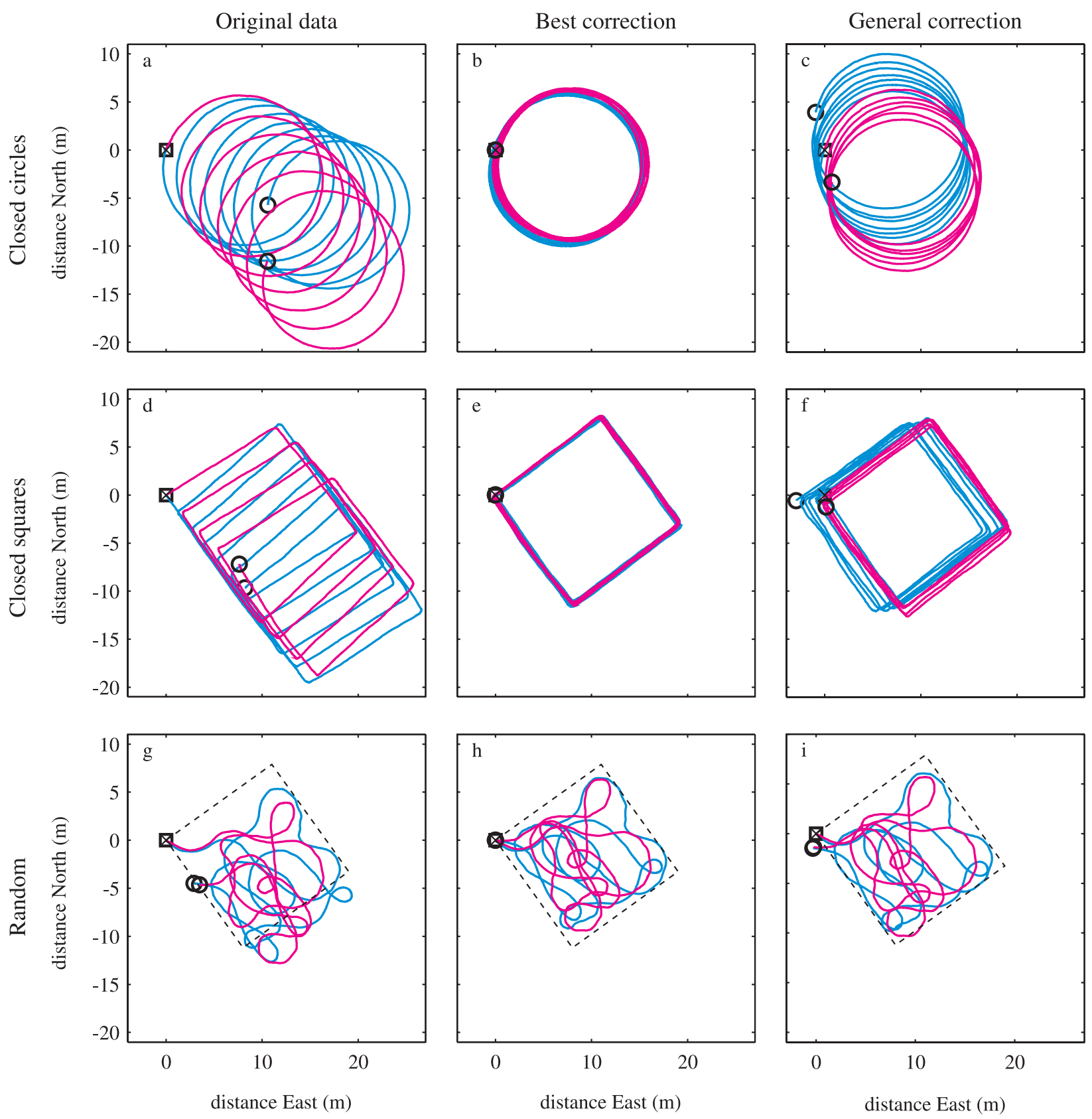

FIG. 3. Plots of original and corrected (best and general) positions for the series of test cases: circles, squares, and random swimming. In each panel, two tracks are shown. For the circle and square cases, blue is the counterclockwise track and red is the clockwise track, whereas the random swimming panels show two representative tracks. Dashed lines in random swimming panels denote the box the diver stayed within during the surveys. Starting points are denoted by a square symbols and endpoints are open circles.

highly repeatable (Fig. 2), indicating that they were not due to random operator error. Thus, the results of the first test suggest there may be significant uncertainty in the direction measured by the compass.

In the second and third tests, when the diver was swimming clockwise and counterclockwise circles and squares, there was a general southeastward drift in the position recorded by the Cobra-Tac (Figs. 3a,b; note the corrected paths also shown in the figure are described in detail below). The along-track pathlengths were correct to within measurable limits; for example, the lengths of the sides of the square were correct, indicating that the average bottom-track speed of the DVL over each side was accurate. However, the angles between adjacent 
sides of the squares were not $90^{\circ}$, again suggesting the cause of the position drift was related to an error in the compass directional measurement. The final test, random swimming within a square marked with ground lines, resulted in apparent excursions outside of the box and an overall drift in a southeastward direction (Fig. 3c).

For the tests conducted over the patch reef (rough bottom), the Cobra-Tac lost bottom track for brief periods (ranging from 0.2 to $2 \mathrm{~s}$ ) and recorded dead reckoning positions showed large jumps (data not shown). Screening the data for anomalously large bottom-track velocities during postprocessing and replacing them with dead reckoning velocities from the last good fix until bottom track was reestablished resulted in very similar paths to those shown in Figs. 3a,b for the sandy bottom tests. Depending on the turn rate and speed changes, the loss of bottom track can significantly degrade navigation results but do not explain the systematic navigation drift described above.

\section{b. One-cycle compass error theory}

Compass error was identified as the most likely cause of the systematic drifts in position measurements during the initial tests. Error in compass measurements arises from imperfect calibration or the presence of an additional magnetic field in the calibration area or at the field site. Compass error is therefore typically a function of heading. Because the origin of this compass error is external magnetic forces, the error is well represented by a one-cycle (hard iron) model (Denne 1998)

$$
\Gamma=A+B \sin \theta+C \cos \theta,
$$

where $\Gamma$ is the heading-dependent compass error, $\theta$ is the compass heading recorded by the instrument, $A$ is the constant heading offset (e.g., sum of the local magnetic declination and beam 3 misalignment), $B$ is the fore-aft permanent (or hard iron) magnetic field, and $C$ is the starboard-port permanent magnetic field. An improved representation of compass error is the two-cycle (hard and soft iron) model

$$
\Gamma=A+B \sin \theta+C \cos \theta+D \cos 2 \theta+E \sin 2 \theta,
$$

where the additional terms represent induced magnetism. The coefficient $D$ represents induced magnetism from symmetrical soft iron and $E$ from asymmetric soft iron (Denne 1998).

Previous studies that have used one- and two-cycle error models to estimate and correct heading errors have determined true headings from gyrocompass or GPS readings (e.g., Munchow et al. 1995). In those studies, the error in each compass measurement was computed from the difference between compass and GPS heading measurements and coefficients were determined by fitting (1) to the measured compass errors. For diver-operated underwater systems such as the Cobra-Tac, a GPS cannot be used. As the true heading and position at any point in time is unknown, a different procedure for estimating the coefficients in (1) is required.

\section{c. Estimation of one-cycle error model coefficients}

In Eq. (1), $A$ is a known constant equal to the sum of the local magnetic declination and the beam 3 offset. As this coefficient results in a simple solid-body rotation, it is assumed that headings have already been corrected by this constant value. Here, we derive an expression for $B$ and $C$, the one-cycle model coefficients, for any path beginning and ending at the same position. The tests described above showed that the average speed for each leg of every path (perpendicular lines, squares, and circles) was correct, indicating that instantaneous speed measurements were not biased and that speed errors were not directionally dependent. To estimate the one-cycle model coefficients, we assume that instantaneous DVL bottom-track speed measurements are correct, and that position error is due to the compass error alone. We also assume that the effect of pitch and roll on compass heading error is negligible, as found by Alderson and Cunningham (1999). The effect of pitch and roll is discussed further in section $3 \mathrm{e}$.

Let $u_{i}, \theta_{i}$, and $\alpha_{i}$ be the bottom-track speed, reported instrument compass heading, and angle between the bottom-track direction and the instrument heading at time $t_{i}$, respectively; and let $\Delta t_{i}$ be the time between consecutive DVL measurements. The uncorrected position $(x, y)$ at any time $t_{m}$ is

$$
\begin{aligned}
& x\left(t_{m}\right)=x_{0}+\sum_{i=1}^{m} u_{i} \Delta t_{i} \sin \left(\theta_{i}+\alpha_{i}\right), \\
& y\left(t_{m}\right)=y_{0}+\sum_{i=1}^{m} u_{i} \Delta t_{i} \cos \left(\theta_{i}+\alpha_{i}\right) .
\end{aligned}
$$

Let $s$ be the total distance between the start $\left(x_{0}, y_{0}\right)$ and the uncorrected end $\left(x_{N}, y_{N}\right)$ positions and let $\phi$ be the heading from $\left(x_{0}, y_{0}\right)$ to $\left(x_{N}, y_{N}\right)$ (Fig. 4). From (3), it follows that

$$
\begin{aligned}
& \sum_{i=1}^{N} u_{i} \Delta t_{i} \sin \left(\theta_{i}+\alpha_{i}\right)=s \sin \phi \\
& \sum_{i=1}^{N} u_{i} \Delta t_{i} \cos \left(\theta_{i}+\alpha_{i}\right)=s \cos \phi
\end{aligned}
$$




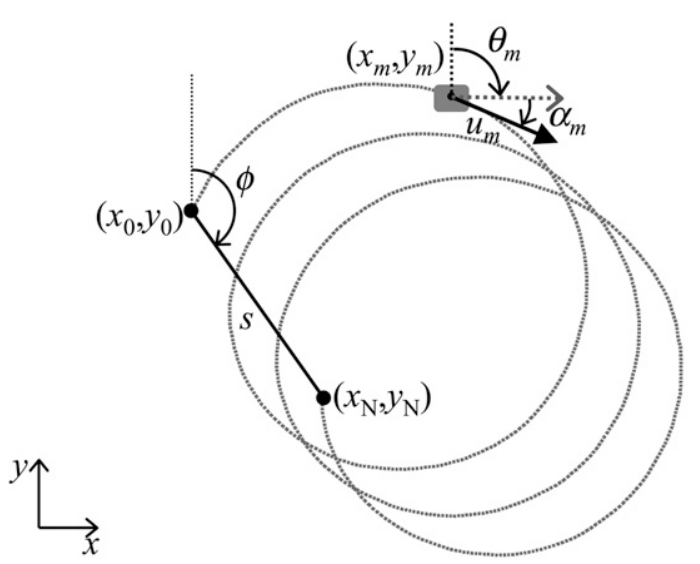

North

$\widehat{\bigsqcup}$

FIG. 4. Schematic diagram illustrating the geometry and symbols used in the correction procedure. Instantaneous Cobra-Tac location $\left(x_{m}, y_{m}\right)$ is indicated by the gray shaded box. Starting location is $\left(x_{0}, y_{0}\right)$, and the final location determined from uncorrected navigation is $\left(x_{N}, y_{N}\right)$. The offset distance is $s$ and offset heading is $\phi$. The Cobra-Tac instantaneous speed is $u_{m}$, uncorrected heading $\theta_{m}$, and angle between instrument heading and direction of travel $\alpha_{m}$.

If $\gamma_{i}=\gamma\left(\theta_{i}\right)$ is the heading-dependent compass error, the true position $\left(x^{\prime}, y^{\prime}\right)$ at time $t_{m}$ is

$$
\begin{aligned}
& x^{\prime}\left(t_{m}\right)=x_{0}+\sum_{i=1}^{m} u_{i} \Delta t_{i} \sin \left(\theta_{i}-\gamma_{i}+\alpha_{i}\right), \\
& y^{\prime}\left(t_{m}\right)=y_{0}+\sum_{i=1}^{m} u_{i} \Delta t_{i} \cos \left(\theta_{i}-\gamma_{i}+\alpha_{i}\right) .
\end{aligned}
$$

Because the actual start and endpoints are the same, $x^{\prime}\left(t_{N}\right)=x_{0}$ and $y^{\prime}\left(t_{N}\right)=y_{0}$, then

$$
\begin{aligned}
& \sum_{i=1}^{N} u_{i} \Delta t_{i} \sin \left(\theta_{i}-\gamma_{i}+\alpha_{i}\right)=0 \\
& \sum_{i=1}^{N} u_{i} \Delta t_{i} \cos \left(\theta_{i}-\gamma_{i}+\alpha_{i}\right)=0 .
\end{aligned}
$$

Using trigonometric identities to expand the angle summation and applying the small angle approximations $\sin \gamma \approx \gamma$ and $\cos \gamma \approx 1$ yields

$$
\begin{aligned}
& \sum_{i=1}^{N} u_{i} \Delta t_{i}\left[\sin \left(\theta_{i}+\alpha_{i}\right)-\gamma_{i} \cos \left(\theta_{i}+\alpha_{i}\right)\right]=0 \\
& \sum_{i=1}^{N} u_{i} \Delta t_{i}\left[\cos \left(\theta_{i}+\alpha_{i}\right)+\gamma_{i} \sin \left(\theta_{i}+\alpha_{i}\right)\right]=0 .
\end{aligned}
$$

The first terms in (7) can be simplified by substituting into (4), then

$$
\begin{aligned}
& \sum_{i=1}^{N} u_{i} \Delta t_{i} \gamma_{i} \cos \left(\theta_{i}+\alpha_{i}\right)=s \sin \phi \\
& \sum_{i=1}^{N} u_{i} \Delta t_{i} \gamma_{i} \sin \left(\theta_{i}+\alpha_{i}\right)=-s \cos \phi .
\end{aligned}
$$

Expressing the compass error $\gamma_{i}$ using the standard onecycle model

$$
\gamma_{i}=B \sin \theta_{i}+C \cos \theta_{i}
$$

where $B$ and $C$ are the coefficients to be determined. Substituting (9) into (8) yields

$$
\begin{aligned}
& B \sum_{i=1}^{N} u_{i} \Delta t_{i} \sin \theta_{i} \cos \left(\theta_{i}+\alpha_{i}\right) \\
& \quad+C \sum_{i=1}^{N} u_{i} \Delta t_{i} \cos \theta_{i} \cos \left(\theta_{i}+\alpha_{i}\right)=s \sin \phi \\
& B \sum_{i=1}^{N} u_{i} \Delta t_{i} \sin \theta_{i} \sin \left(\theta_{i}+\alpha_{i}\right) \\
& \quad+C \sum_{i=1}^{N} u_{i} \Delta t_{i} \cos \theta_{i} \sin \left(\theta_{i}+\alpha_{i}\right)=-s \cos \phi
\end{aligned}
$$

This set of equations can be solved simultaneously to give expressions for $B$ and $C$ :

$$
\begin{aligned}
& B=\frac{-s\left(M_{12} \cos \phi+M_{22} \sin \phi\right)}{M_{12} M_{21}-M_{11} M_{22}} \\
& C=\frac{s\left(M_{11} \cos \phi+M_{21} \sin \phi\right)}{M_{12} M_{21}-M_{11} M_{22}},
\end{aligned}
$$

where

$$
\begin{aligned}
& M_{11}=\sum_{i=1}^{N} u_{i} \Delta t_{i} \sin \theta_{i} \cos \left(\theta_{i}+\alpha_{i}\right) \\
& M_{12}=\sum_{i=1}^{N} u_{i} \Delta t_{i} \cos \theta_{i} \cos \left(\theta_{i}+\alpha_{i}\right) \\
& M_{21}=\sum_{i=1}^{N} u_{i} \Delta t_{i} \sin \theta_{i} \sin \left(\theta_{i}+\alpha_{i}\right) \\
& M_{22}=\sum_{i=1}^{N} u_{i} \Delta t_{i} \cos \theta_{i} \sin \left(\theta_{i}+\alpha_{i}\right) .
\end{aligned}
$$

Thus, the one-cycle model coefficients $B$ and $C$ can be obtained for any path that begins and ends at the same position with a knowledge of the DVL bottom-track speed, uncorrected heading, time between measurements, and the apparent offset distance and heading between 


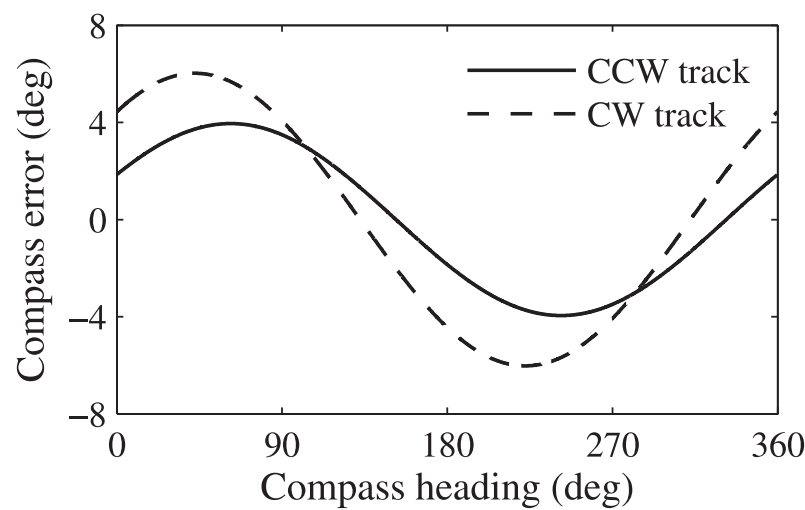

FIG. 5. One-cycle compass error $\gamma$ as a function of heading for the closed circle tests shown in Fig. 3.

start and end points. The highest-quality correction curve will be obtained if all headings are sampled equally during the diver path. Assuming that there will be some variability in the diver's path, repeated continuous circuits around a circular path (as in test 2) will yield the best estimates of the one-cycle model coefficients.

\section{d. Application of correction methods}

The correction method derived in the previous section was applied to the Cobra-Tac position measurements described above. When the correction method was applied to each test case separately, using the measurements from that test case, near-perfect correction was achieved (Figs. 3d-f). However, the correction coefficients derived for each test case were different. Additionally, the correction curves $\gamma(\theta)$ derived for the counterclockwise swimming paths were significantly different than those for the clockwise swimming paths (Fig. 5).

A correction method that can be implemented before beginning navigation or mapping is desired. To this end, a "general" correction curve was computed as the mean of the correction curves for counterclockwise and clockwise circular paths, and this correction was applied to each of the test cases (Figs. 3g-i). Although the general correction significantly improved the test paths, an obvious directional bias remained in all cases. The correction curves appear to differ depending on whether the diver makes more frequent left- or right-hand turns. There are several possible explanations for this discrepancy. One possibility is that the diver held the Cobra-Tac in a slightly different position relative to his dive equipment (which included a stainless steel back plate and tank bands), depending on the swim direction. To determine whether this was the case, the above tests were repeated using an entirely nonferrous recreational dive rig. Very similar results were obtained, indicating that the dive equipment used in the original tests was not the primary cause of compass heading error. Another possible explanation is that position error differed depending on whether the diver swam into or with the ambient current. During the tests, the current was $0.1 \mathrm{~m} \mathrm{~s}^{-1}$ toward the northeast, yet the drift was in a southeasterly direction. Additionally, an analysis, shown below, indicates that for an ambient current of this magnitude the along-track uncertainty associated with water speed relative to the DVL is small compared with an across-track uncertainty due to compass error (see section 3e). A third possibility is that the one-cycle compass error model does not adequately capture the heading dependence of the compass error. A two-cycle compass correction, as defined in (2), was also implemented and resulted in very little improvement over the one-cycle correction. Finally, compass error may also depend on instrument tilt, and this is examined in the following section.

\section{e. Analysis of uncertainties}

A systematic analysis of error in Cobra-Tac position measurements is required to compare its field performance with the manufacturer's specifications and to evaluate the effectiveness of the correction procedure developed above. Teledyne RD Instruments (2007) gives the along-track accuracy as $0.2 \%$ of the along-track water velocity relative to the instrument (the sum of ambient current and the instrument velocity over bottom), plus inherent static drift in the DVL. The maximum expected along-track uncertainty at the end of a diver path (time $t_{N}$ ) can therefore be expressed as

$$
E_{\mathrm{TRDI}, \mathrm{L}}=\sum_{i=1}^{N-1}\left\{\left[0.002\left(U_{\mathrm{amb}}+u_{i}\right) \Delta t_{i}\right]^{2}+\left(u_{\mathrm{static}} \Delta t_{i}\right)^{2}\right\}^{1 / 2}
$$

where $E_{\mathrm{TRDI}, \mathrm{L}}$ is the along-track uncertainty in meters, $U_{\text {amb }}$ is the ambient current speed, $u_{i}$ is the bottom-track speed relative to the bottom, and $\Delta t_{i}$ is the time interval between consecutive pings. DVL static drift $u_{\text {static }}$ approaches zero as the instrument's velocity approaches zero (Teledyne RD Instruments 2009, personal communication), but a constant value of $0.001 \mathrm{~m} \mathrm{~s}^{-1}$ was used as an upper-bound estimate of this error source. The ambient current during the field tests (from a nearby acoustic Doppler profiler) was a maximum of $0.1 \mathrm{~m} \mathrm{~s}^{-1}$ and the diver swimming speed averaged $0.35 \mathrm{~m} \mathrm{~s}^{-1}$, thus the two terms in Eq. (13) are comparable in size and the along-track uncertainty is at most $0.4 \%$ of the along-track distance. The along-track uncertainty is due solely to the uncertainty in the DVL bottom-track velocity, whereas the across-track uncertainty accounts for compass accuracy. The manufacturer-specified across-track accuracy is 
TABLE 1. Navigation error $\boldsymbol{\varepsilon}_{N}$ for CC and CW test cases before and after correction. Errors are expressed as a percentage of total track length. The maximum expected uncertainty based on manufacturer specifications is $4 \%$. Navigation errors larger than the manufacturer specifications are italicized.

\begin{tabular}{|c|c|c|c|c|}
\hline & \multicolumn{2}{|c|}{ Original } & \multicolumn{2}{|c|}{ Corrected } \\
\hline & $\mathrm{CC}$ & $\mathrm{CW}$ & $\mathrm{CC}$ & $\mathrm{CW}$ \\
\hline \multicolumn{5}{|c|}{ Conch Reef tests } \\
\hline Circles & 3.5 & 5.3 & 1.2 & 1.2 \\
\hline Squares & 3.8 & 4.7 & 0.9 & 0.5 \\
\hline \multirow[t]{3}{*}{ Random } & \multicolumn{2}{|c|}{4.0} & \multicolumn{2}{|c|}{1.1} \\
\hline & \multicolumn{2}{|c|}{4.5} & \multicolumn{2}{|c|}{1.1} \\
\hline & \multicolumn{2}{|c|}{ orea tests } & & \\
\hline Level, $1 \mathrm{~m}$ above bottom & 1.5 & 1.5 & 0.3 & 0.3 \\
\hline Increased pitch & 2.0 & 1.5 & 0.9 & 0.9 \\
\hline Increased roll & 1.6 & 1.8 & 0.3 & 0.3 \\
\hline Increased height & 1.2 & 1.4 & 0.4 & 0.4 \\
\hline \multirow[t]{3}{*}{ In situ calibration } & 1.1 & 1.1 & 0.2 & 0.2 \\
\hline & 1.2 & 1.0 & 0.2 & 0.2 \\
\hline & 0.9 & 0.7 & 0.3 & 0.3 \\
\hline
\end{tabular}

$3.5 \%$ of the along-track distance. The expected acrosstrack uncertainty at the end of a diver path is therefore

$$
E_{\mathrm{TRDI}, \mathrm{C}}=0.035 \sum_{i=1}^{N-1} u_{i} \Delta t_{i}
$$

As the paths in each test case involved many turns and sampled different headings, it is reasonable to expect the across-track uncertainty, which is the larger of the two manufacturer specifications and depends on compass accuracy, to dominate. Thus, the maximum expected fractional error according to the manufacturer specifications is about $4 \%$.

To enable comparison of measured errors with accuracies specified by the manufacturer, the final measurement error $E_{N}$ is defined as the distance between the position at the end of the track according to the CobraTac $\left(x_{N}, y_{N}\right)$ and the true position at the end of the track $\left(x_{N}^{\prime}, y_{N}^{\prime}\right)$, which was equal to the start position $\left(x_{0}, y_{0}\right)$ for all test cases. The fractional error $\boldsymbol{\varepsilon}_{N}$ is defined as the position error $E_{N}$ divided by the total pathlength, that is,

$$
\varepsilon_{N}=\frac{E_{N}}{\sum_{i=1}^{N-1} u_{i} \Delta t_{i}}=\frac{\left[\left(x_{N}-x_{N}^{\prime}\right)^{2}+\left(y_{N}-y_{N}^{\prime}\right)^{2}\right]^{1 / 2}}{\sum_{i=1}^{N-1} u_{i} \Delta t_{i}} .
$$

The original (uncorrected) fractional error and fractional error after general correction for each of the tests are given in Table 1. Using the best correction, navigation error was reduced to zero because of the way in which the error was computed. For the field tests at Conch Reef, navigation errors for all test paths were
$90 \%-130 \%$ of the expected accuracy. Using the general correction derived from clockwise and counterclockwise circular tracks, the navigation error was reduced to well within the manufacturer-specified accuracy. For the corresponding field test in Moorea (level instrument at $1 \mathrm{~m}$ above bottom), the navigation errors were $40 \%$ of the expected accuracy.

Navigation errors for the tests at Conch Reef were 23 times larger than those for the tests in Moorea. Conch Reef is the site of the National Oceanic and Atmospheric Administration's (NOAA) Aquarius Reef Base; their underwater habitat was located about $30-40 \mathrm{~m}$ from the Cobra-Tac test site and a set of four large steel air flasks was $10-20 \mathrm{~m}$ from the test site. The larger errors in the tests at Conch Reef are thought to arise because of these nearby ferromagnetic materials. Although the amount of ferromagnetic material was unusually large at Conch Reef, the results illustrate that large navigation error (more than $5 \%$ of the track length) can be introduced by local magnetic fields not accounted for in the bench calibration.

The navigation error at any point along the diver's path is defined as the distance between the position at time $t_{m}$ according to the Cobra-Tac $\left(x_{m}, y_{m}\right)$ and the true position $\left(x_{m}^{\prime}, y_{m}^{\prime}\right)$. The true position at time $t_{m}$ was estimated from the results of the "best correction" procedure described in the previous section. Thus, the navigation error is computed as a function of along-track distance using

$$
E_{m}=\left[\left(x_{m}-\hat{x}_{m}^{\prime}\right)^{2}+\left(y_{m}-\hat{y}_{m}^{\prime}\right)^{2}\right]^{1 / 2},
$$

where $\left(\hat{x}_{m}^{\prime}, \hat{y}_{m}^{\prime}\right)$ is the best estimate of the true position at time $t_{m}$.

In an average sense, the navigation error varies linearly with along-track pathlength (Fig. 6). Because the compass error is a function of heading, the navigation error oscillates regularly around the linear average for the uncorrected circular path (Fig. 6a) and in a stepwise fashion for the square path (Fig. 6b). For the random path, the navigation error varies randomly about the linear average (Fig. 6c). For all uncorrected test cases shown, the navigation error is somewhat greater than (lies above) the manufacturer-specified across-track accuracy, and it is much greater than the manufacturer-specified alongtrack accuracy. When the general correction is applied, the navigation error is well within manufacturer-specified accuracy.

Because compass error is the main contributor to CobraTac position error, the most direct measure of accuracy is root-mean-square compass error, as this gives a direct indication of the quality of the compass calibration. The rms compass error, evaluated over all headings, is 

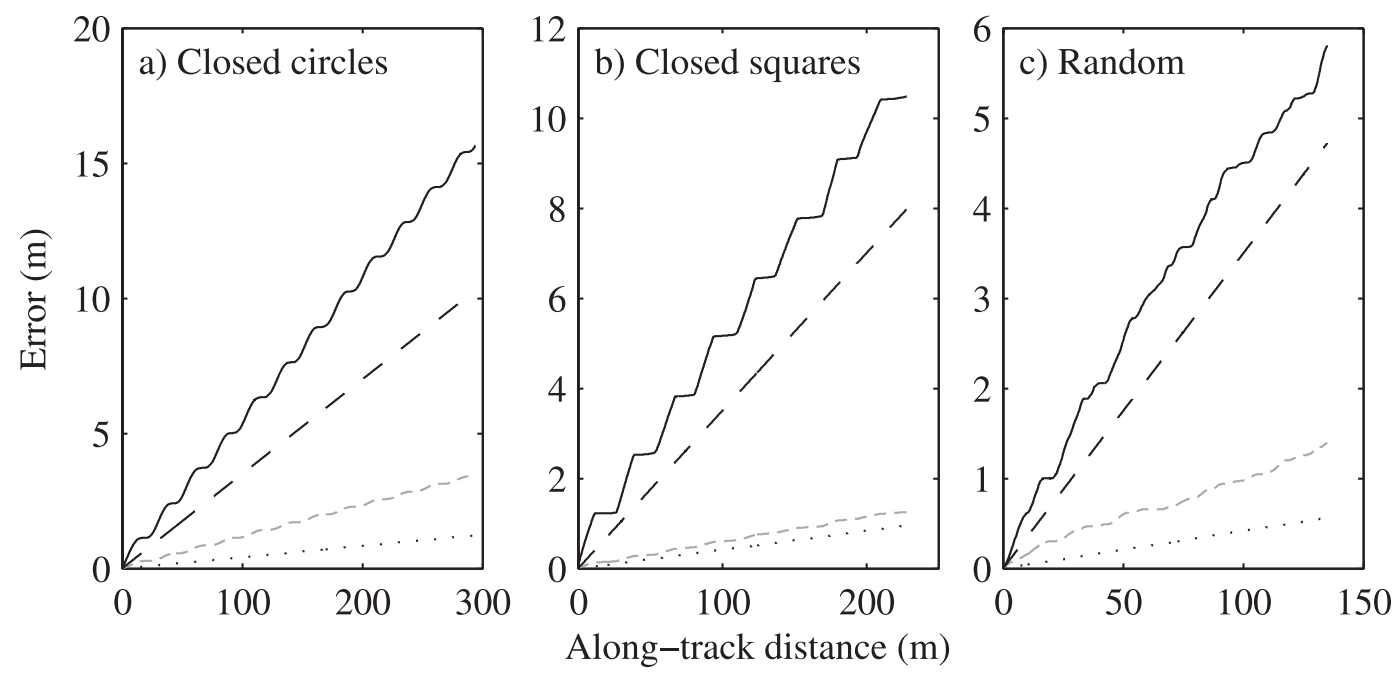

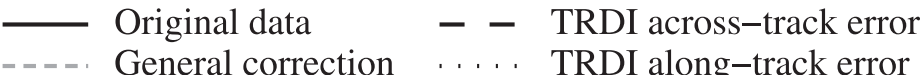

FIG. 6. Cobra-Tac position error vs along-track distance for the three counterclockwise test tracks. Panels show comparisons between measurement errors and along- and across-track accuracy specifications from Teledyne RD Instruments.

$$
\begin{aligned}
E_{\theta} & =\left\{\frac{1}{2 \pi} \int_{0}^{2 \pi}[\gamma(\theta)]^{2} d \theta\right\}^{1 / 2} \\
& =\left[\frac{1}{2 \pi} \int_{0}^{2 \pi}(B \sin \theta+C \cos \theta)^{2} d \theta\right]^{1 / 2}=\left(\frac{B^{2}+C^{2}}{2}\right)^{1 / 2}
\end{aligned}
$$

As seen in Fig. 5, the directional dependence of compass error was different for clockwise and counterclockwise paths. The rms difference between heading error for clockwise and counterclockwise paths is given by

$$
E_{\theta, \mathrm{cc}-\mathrm{cw}}=\left[\frac{\left(B_{\mathrm{cc}}-B_{\mathrm{cw}}\right)^{2}+\left(C_{\mathrm{cc}}-C_{\mathrm{cw}}\right)^{2}}{2}\right]^{1 / 2} .
$$

Thus, $E_{\theta, \mathrm{cc}-\mathrm{cw}}$ is an indicator of how good the general correction procedure will be.

Root-mean-square compass errors were much larger for the tests at Conch Reef than for the tests in Moorea, most likely due to the presence of ferromagnetic material at Conch Reef (Table 2). Although rms compass error was not significantly affected by instrument tilt, the difference between the compass errors for $\mathrm{CC}$ and $\mathrm{CW}$ paths was larger when the instrument was deliberately tilted in the direction of motion (pitch). As the general correction uses an average of the correction curves for clockwise and counterclockwise paths, it performs poorly if the instrument is not held level, particularly along the axis of motion (Table 1). Instrument height above bottom had little effect, as tests repeated at $2 \mathrm{~m}$ above bottom had similar error as those at $1 \mathrm{~m}$ above bottom (Table 1 ).

Tests that used the new firmware version with in situ compass underwater calibration had smaller rms compass error (less than $1^{\circ}$ ) than those with the standard bench compass calibration (Table 2). This translated into significantly reduced navigation errors (Table 1 ). With the in situ compass calibration, uncorrected navigation errors

TABLE 2. Pitch, roll, and rms heading-dependent compass error $E_{\theta}$ for CC and CW test cases and for the difference between CC and $\mathrm{CW}$ compass errors. All quantities are in degrees. Pitch and roll are reported as the mean \pm 1 standard deviation.

\begin{tabular}{lccccc}
\hline \hline & \multicolumn{5}{c}{$E_{\theta}$} \\
\cline { 4 - 5 } & Pitch & Roll & CC & CW & $E_{\theta, \mathrm{cc}-\mathrm{cw}}$ \\
\hline \multicolumn{5}{c}{ Conch Reef tests } \\
Circles & $-4.1 \pm 2.6$ & $-0.3 \pm 2.0$ & 2.8 & 4.3 & 1.9 \\
Squares & $-2.2 \pm 2.5$ & $2.0 \pm 1.7$ & 3.1 & 3.8 & 0.8 \\
Random & $-2.8 \pm 2.2$ & $0.4 \pm 2.6$ & 4.0 & N/A \\
& $-1.2 \pm 2.1$ & $0.5 \pm 2.7$ & 4.0 & N/A \\
Level, 1 m. a. b. & $-4.1 \pm 2.6$ & $0.8 \pm 2.3$ & 1.2 & 1.2 & 0.5 \\
Increased pitch & $-15.5 \pm 2.4$ & $0.4 \pm 2.4$ & 1.6 & 1.2 & 1.4 \\
Increased roll & $-6.5 \pm 2.6$ & $13.4 \pm 2.5$ & 1.3 & 1.4 & 0.5 \\
Increased height & $-3.5 \pm 2.8$ & $-0.9 \pm 2.7$ & 1.0 & 1.1 & 0.6 \\
In situ calibration & $-3.0 \pm 2.6$ & $-0.5 \pm 2.5$ & 0.9 & 0.9 & 0.3 \\
& $-2.8 \pm 2.5$ & $-0.7 \pm 2.7$ & 0.9 & 0.8 & 0.4 \\
& $-3.5 \pm 2.3$ & $-1.0 \pm 2.4$ & 0.8 & 0.6 & 0.4 \\
\hline
\end{tabular}


were close to $1 \%$ of the track length, and after the general correction was applied navigation errors were $0.2-0.3 \%$ of the track length, a factor of 10 better than manufacturer specifications.

\section{Conclusions}

Cobra-Tac navigation data from tests at Conch Reef, Florida, had an obvious directional bias that was greater than the error expected from manufacturer specifications. Systematic field tests showed that the DVL speed measurements were accurate but that positional error could be as large as $5 \%$ of the track length due to directionally dependent compass error. Navigation errors of this magnitude could result in not finding an instrument after a long swim in low visibility, or large errors in maps or surveys. Much of this error can be attributed to the presence of ferromagnetic material at a field site, and the tests and analyses developed herein illustrate the importance of obtaining a compass calibration that mimics field conditions as closely as possible. A postprocessing procedure was derived, based on a one-cycle compass error model, to correct Cobra-Tac positions. The correction coefficients differed between clockwise (CW) and counterclockwise (CC) test paths. A general correction based on the average of $\mathrm{CW}$ and $\mathrm{CC}$ coefficients improved position measurements to within about $1 \%$ of the track length, significantly better than manufacturer specifications. The difference between heading-dependent compass errors for $\mathrm{CW}$ and $\mathrm{CC}$ paths was shown to be associated with nonzero instrument tilt in the direction of motion. Thus, operator skill is important for obtaining good navigation data from a Cobra-Tac.

The results suggest that before using a Cobra-Tac to make position measurements it is useful to obtain an in situ dataset consisting of repeated circular swims in a CW path followed by repeated circular swims in CC path. These data can then be used for postdeployment uncertainty analysis (to assess uncertainty associated with bottom topography and diver skill) and postdeployment position correction to account for local magnetic fields that are not present during predeployment compass calibration. The correction is straightforward to implement and uses only information that is recorded internally during normal operation of the instrument. Because the correction is a postprocessing step, it will improve the capability of Cobra-Tac for many scientific applications (e.g., mapping). However, it cannot be used to correct positions displayed by the instrument in real time.

In response to the initial tests, Teledyne RD Instruments provided a new firmware version that allowed in situ underwater field calibration of the compass by a diver. Tests using the new firmware indicate that with in situ compass calibration, the error in Cobra-Tac position measurements is about $1 \%$ of the pathlength, well within manufacturer specifications. This level of accuracy should be sufficient for a wide range of scientific applications.

Acknowledgments. We thank Omer Poroy, Greg Rivalan, and Vadim Polonichko at Teledyne RD Instruments for generously arranging Cobra-Tac training and instrument loans. Dale Stokes and Mark Reagan gave helpful advice on operating the Cobra-Tac. Patrick Gibson and Sarah Giddings provided assistance in the field. We also thank the entire staff of the National Undersea Research Center, Key Largo, for field support during an Aquarius saturation diving mission where most of the data were collected. Three anonymous reviewers provided comments that significantly improved the manuscript. Partial funding for this work was provided by NSF (OCE-0417412; OCE-0622967) and the NOAA National Undersea Research Program (SEGM-2006-03-01).

\section{REFERENCES}

Alderson, S. G., and S. A. Cunningham, 1999: Velocity errors in acoustic Doppler current profiler measurements due to platform attitude variations and their effect on volume transport estimates. J. Atmos. Oceanic Technol., 16, 96-106.

Denne, W., 1998: Magnetic Compass Deviation and Correction. 3rd ed. Brown, Son, and Ferguson, $165 \mathrm{pp}$.

Fong, D. A., and S. G. Monismith, 2004: Evaluation of the accuracy of a ship-mounted, bottom-tracking ADCP in a near-shore coastal flow. J. Atmos. Oceanic Technol., 21, 1121-1128.

Griffiths, G., 1994: Using 3DF GPS heading for improving underway ADCP data. J. Atmos. Oceanic Technol., 11, 11351143.

Joiner, J. T., Ed., 2001: NOAA Diving Manual: Diving for Science and Technology. 4th ed. Best Publishing Co., 660 pp.

Joyce, T. M., 1989: On in situ "calibration" of shipboard ADCPs. J. Atmos. Oceanic Technol., 6, 169-172.

Merifield, P. M., and D. M. Rosencrantz, 1966: A simple method for surveying a small area underwater. Limnol. Oceanogr., 11, 408-409.

Moline, M. A., and Coauthors, 2005: Remote environmental monitoring units: An autonomous vehicle for characterizing coastal environments. J. Atmos. Oceanic Technol., 22, 1797-1808.

Munchow, A., C. S. Coughran, M. C. Hendershott, and C. D. Winant, 1995: Performance and calibration of an acoustic Doppler current profiler. J. Atmos. Oceanic Technol., 12, 435-444.

Newborough, D., and B. Woodward, 1999: Diver navigation and tracking system. Proc. Oceans '99 MTS/IEEE, Vol. 3, Seattle, WA, Institute of Electrical and Electronics Engineers, 15811586.

Teledyne RD Instruments, 2007: Cobra-Tac autonomous navigation and mapping console user's guide. Teledyne Technologies, Inc. P/N 956-6000-00, 56 pp.

Trump, C. L., and G. O. Marmorino, 1997: Calibrating a gyrocompass using ADCP and DGPS data. J. Atmos. Oceanic Technol., 14, 211-214. 